\title{
Narrative review of urinary glycan biomarkers in prostate cancer
}

\author{
Shingo Hatakeyama ${ }^{1 \wedge}$, Tohru Yoneyama $^{2}$, Yuki Tobisawa ${ }^{3} \wedge$, Hayato Yamamoto $^{3}$, Chikara Ohyama $^{1,2,3 \wedge}$ \\ ${ }^{1}$ Department of Advanced Blood Purification Therapy, Hirosaki University Graduate School of Medicine, Hirosaki, Japan; ${ }^{2}$ Department of \\ Glycotechnology, Center for Advanced Medical Research, Hirosaki University Graduate School of Medicine, Hirosaki, Japan; ${ }^{3}$ Department of \\ Urology, Hirosaki University Graduate School of Medicine, Hirosaki, Japan \\ Contributions: (I) Conception and design: S Hatakeyama; (II) Administrative support: None; (III) Provision of study materials or patients: None; \\ (IV) Collection and assembly of data: All authors; (V) Data analysis and interpretation: All authors; (VI) Manuscript writing: All authors; (VII) Final \\ approval of manuscript: All authors. \\ Correspondence to: Shingo Hatakeyama, MD. Department of Urology, Hirosaki University Graduate School of Medicine, 5 Zaifu-cho, Hirosaki 036- \\ 8562, Japan. Email: shingoh@hirosaki-u.ac.jp.
}

\begin{abstract}
Prostate cancer (PC) is the second most common cancer in men worldwide. The application of the prostate-specific antigen (PSA) test has improved the diagnosis and treatment of PC. However, the PSA test has become associated with overdiagnosis and overtreatment. Therefore, there is an unmet need for novel diagnostic, prognostic, and predictive biomarkers of PC. Urinary glycoproteins and exosomes are a potential source of PC glycan biomarkers. Urinary glycan profiling can provide noninvasive monitoring of tumor heterogeneity and aggressiveness throughout a treatment course. However, urinary glycan profiling is not popular due to technical disadvantages, such as complicated structural analysis that requires specialized expertise. The technological development of glycan analysis is a rapidly advancing field. A lectin-based microarray can detect aberrant glycoproteins in urine, including PSA glycoforms and exosomes. Glycan enrichment beads can enrich the concentration of $N$-linked glycans specifically. Capillary electrophoresis, liquid chromatography-tandem mass spectrometry, and matrix-assisted laser desorption/ionization-time of flight mass spectrometry can detect glycans directory. Many studies suggest potential of urinary glycoproteins, exosomes, and glycosyltransferases as a biomarker of PC. Although further technological challenges remain, urinary glycan analysis is one of the promising approaches for cancer biomarker discovery.
\end{abstract}

Keywords: Prostate cancer (PC); urinary; biomarker; glycan; glycosylation

Submitted Jun 05, 2020. Accepted for publication Aug 25, 2020.

doi: 10.21037/tau-20-964

View this article at: http://dx.doi.org/10.21037/tau-20-964

\section{Introduction}

Prostate cancer (PC) is the second most commonly diagnosed malignancy in men worldwide (1-6). The application of the prostate-specific antigen (PSA) test has greatly improved the diagnosis and treatment of PC (7-24). However, PSA has become associated with the overdiagnosis of patients with the non-aggressive disease and displays limited usefulness in patients with castrationresistant PC $(5,25-37)$. Therefore, there is an unmet need for novel diagnostic (detection of early-stage disease, and differentiation of benign from malignant disease), prognostic (prediction of disease outcome and monitoring

^ ORCID: Shingo Hatakeyama, 0000-0002-0026-4079; Tohru Yoneyama, 0000-0002-1098-407X; Yuki Tobisawa, 0000-0001-8026-9541; Chikara Ohyama, 0000-0003-1550-8379. 
of disease recurrence), and predictive (monitoring of the response to therapeutics and aiding treatment decisions) biomarkers.

Many studies suggested the role of glycans as potential biomarkers for diseases (38-54). A broad range of glycan alterations have been observed in blood-based or tissuebased analysis, including aberrant PSA glycosylation, increased sialylation, core fucosylation, $O$-GlcNAcylation, and branched $N$-glycan formation (55-71). Of fluid-based biomarkers, urine is one of the most minimally invasive and promising sources for the discovery of new biomarkers of PC and contains cells, DNA, RNA, proteins, extracellular vesicles (exosomes), and glycans (72-80). However, not enough evidence currently exists to support the identification of urine-based glycan biomarkers of PC, due to methodological difficulties in analyzing complex glycan structure. However, the technological development of glycan analysis is rapidly advancing in association with the development of high-throughput platforms. In this review, we discuss the overview of glycan analysis and the potential of urinary glycans for diagnostic and prognostic biomarkers for PC. We present the following article in accordance with the Narrative Review reporting checklist (available at http:// dx.doi.org/10.21037/tau-20-964).

\section{Overview of glycan analysis}

\section{Role of glycosylation and types of glycoproteins}

Glycans (saccharides) are known to have crucial roles in molecular communications and are essential for nearly every biological process (Figure 1A) (81). It is believed that over $50 \%$ of all proteins are glycosylated $(40,82)$. Glycosylation is the most common posttranslational modification and is tightly controlled by specific glycosylation enzymes via oneto-one correspondence, the expression of glycosylation enzymes are regulated by epigenetic modification (41). There are two types of glycosylation; $O$-linked and $N$-linked glycosylation (Figure 1B) $(40,82)$. $N$-linked glycosylation occurs at the consensus sequence of asparagine- $\mathrm{X}$-serine/ threonine ( $\mathrm{X}$ is any amino acid except proline) and includes major three $N$-glycan structure types (high-mannose, complex, and hybrid glycans) (40). $O$-linked glycans are usually attached to serine or threonine residues and include eight $O$-GalNAc glycan core structures (cores 1 to 8) (82). The extent of glycosylation depends on the number of glycosylation sites in a protein and the expression of specific glycosylation enzymes within the cells. Dysfunction of glycosylation can abnormally influence homeostasis (41,81,83-85). Therefore, cancer therapies targeting glycans may have the potential to improve diagnosis and treatment outcomes (86).

\section{Potential biomarkers in urine}

The advantages of urine analyses include noninvasive and repeat sampling to identify cancers such as PC or urothelial carcinoma (Figure 2). Urine after prostate massage contains many potential biomarkers for PC, including cells, DNA, RNA, proteins, exosomes, bacteria (microbiome), viruses, and other small molecules (72,75,87-92). Several RNA biomarkers have been used clinically, such as in the urinary PC antigen 3 test (RNA-based urinary marker) (93) and the ExoDx Prostate test (detection of PCA3, ERG, and SPDEF genes in urinary exosomes) (94). However, not many glycanbased urinary biomarkers are available due to the technical difficulties of glycan analysis. A lectin-based microarray can detect aberrant glycoproteins in urine (95), including PSA glycoforms and exosomes (60). Glycan enrichment beads (Sweetblot) can specifically enrich the concentration of $N$-linked glycans (96). Capillary electrophoresis, liquid chromatography-tandem mass spectrometry (LC-MS) (97), and matrix-assisted laser desorption/ionization-time of flight mass spectrometry (MALDI-TOF/MS) can detect glycans directly (44). However, each methodology has its strengths and weaknesses for glycan analysis. The lectinbased assay can detect both glycans and core proteins but needs multiple lectins to detect the specific structure of glycans. Mass spectrometry is mandatory to detect the whole structure of glycans, but not easy to detect core proteins. Therefore, multiple steps are necessary to see an overall picture of glycans. This represents a bottleneck in the technique of glycan analysis.

\section{Methods}

\section{Search methods for identification of studies}

PubMed online database was accessed for research on Aug $10^{\text {th }}, 2020$. Searches were performed using the keywords: "prostate cancer", "urine", and "glycan". Each identified abstract was independently evaluated by two authors. All studies were independently evaluated and selected the consistent criteria such as independent cohort, a proper number of samples and controls, clinically meaningful outcomes, and promising diagnostic/prognostic 
A

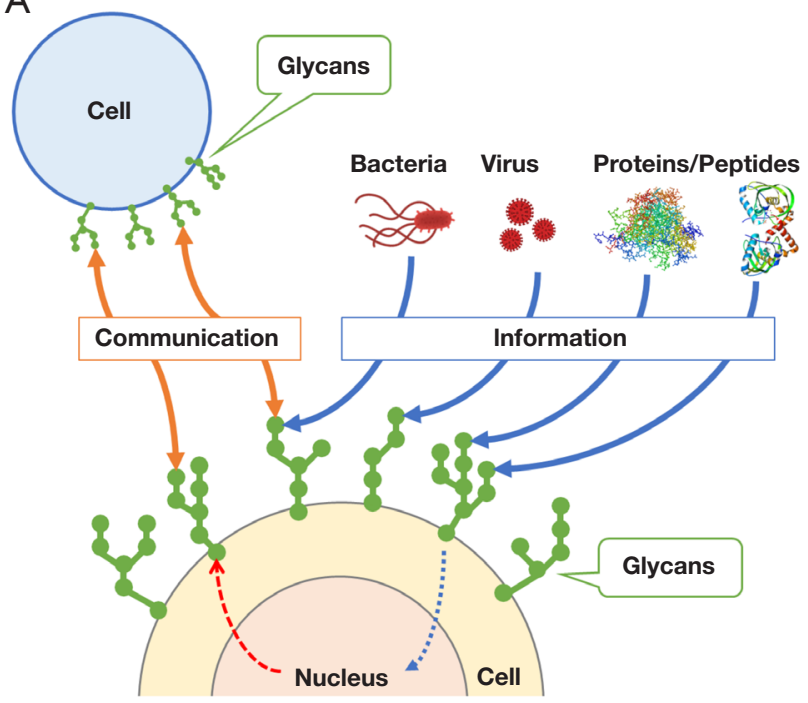

B

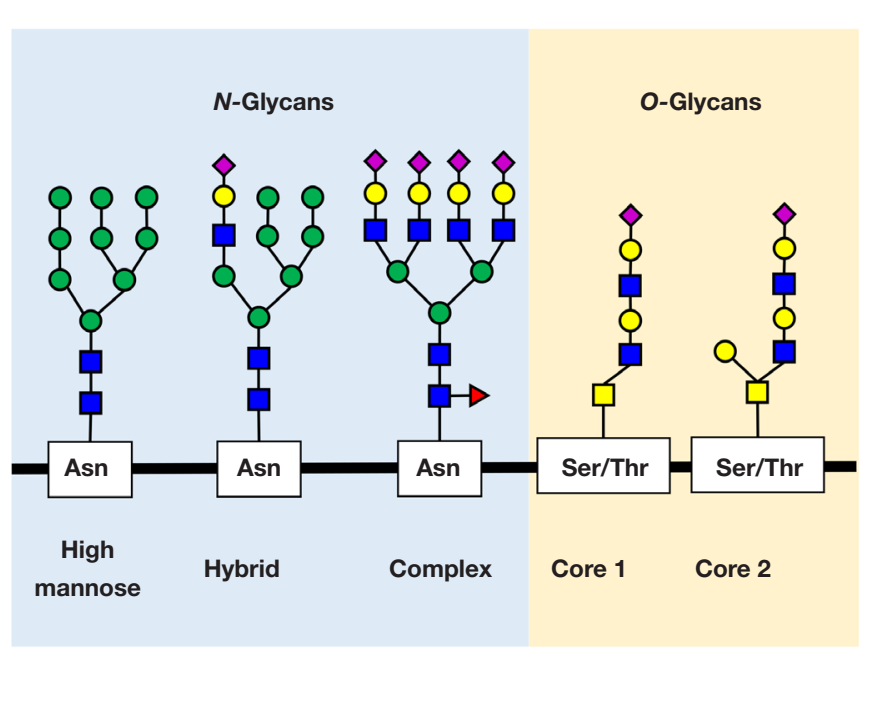

Figure 1 Role and types of glycans. Role of glycans in cell-to-cell communications (A) and types of glycoproteins (B) are shown.

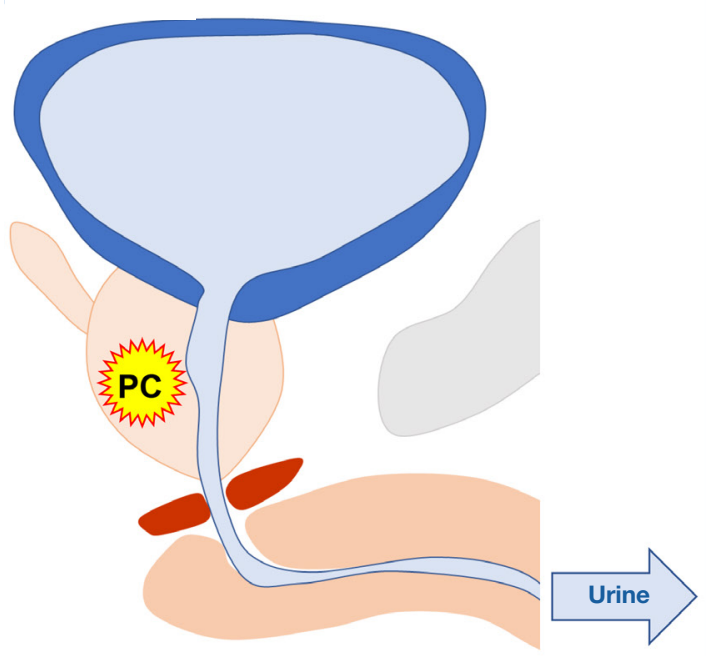

Figure 2 Potential urinary biomarkers for prostate cancer. Urine after prostate massa
including cells, DNA, RNA, proteins, exosomes, bacteria, virus, and other small molecules.
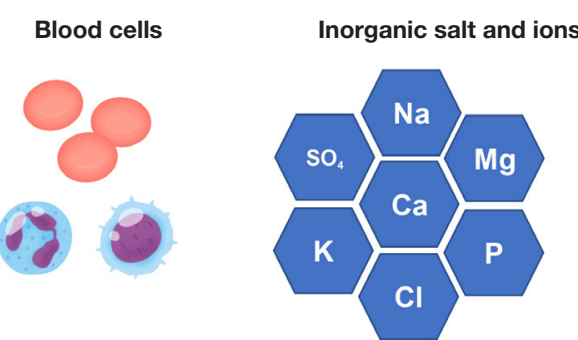

Metabolites

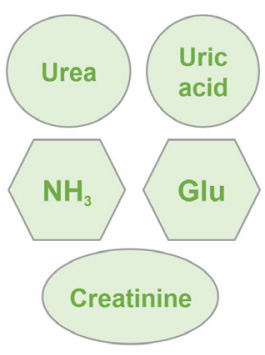

Bacteria/virus

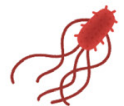

DNA/RNA

Proteins and glycoproteins

$$
\text { 8 }
$$

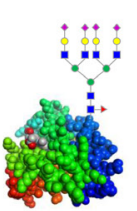

Exosomes

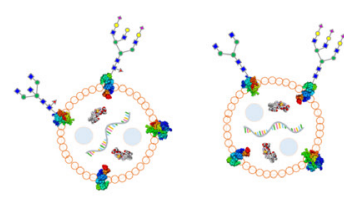

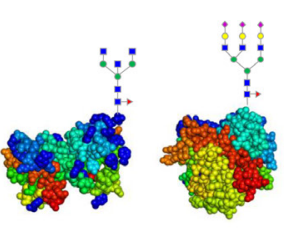

Hyaluronic acid

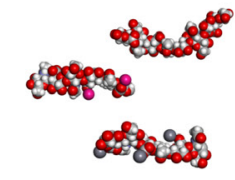




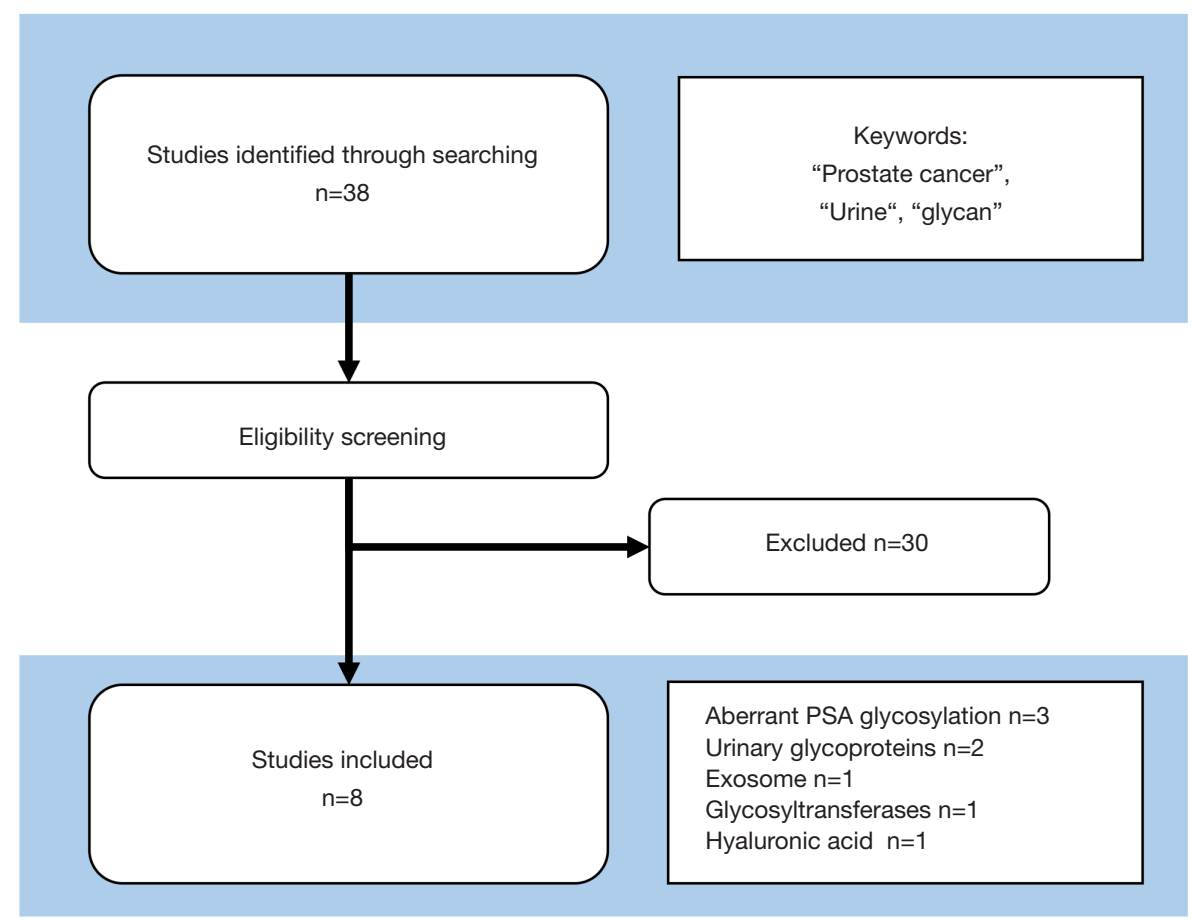

Figure 3 Search methods for identification of studies. PubMed online database was accessed for research on Aug 10th, 2020. Searches were performed using the keywords: "prostate cancer", "urine", and "glycan". Inclusion criteria were (I) independent cohort, (II) a proper number of samples and controls, (III) clinically meaningful outcomes, and (IV) promising diagnostic/prognostic performance.

performance. This study was performed according to the ethical standards of the Declaration of Helsinki and approved by the ethics review boards of the Hirosaki University School of Medicine (authorization number: 2019-001 and 2019-099).

\section{Result of study screening}

We identified 38 studies and excluded 30 studies that did not meet the inclusion criteria. Finally, we included 8 studies in this narrative review (Figure 3). Studies were classified into 5 categories such as (I) aberrant PSA glycosylation, (II) urinary glycoproteins, (III) exosome, (IV) glycosyltransferases, and (V) hyaluronic acid. The number of studies for PC detection, aggressive disease, and both of them were $6(80,97-101), 1$ (77), and 1 (79), respectively.

\section{Urinary glycan analysis detecting aberrant PSA glycosylation}

PSA is a glycoprotein that has been used widely as a biomarker for PC. However, it has been associated with overdiagnosis and overtreatment of non-aggressive cancers $(21,25,35,102)$. There is an unmet clinical need to identify aggressive PC requiring intensive treatment. Several studies have identified specific cancer-associated glycan structures and PSA glycosylation $(63,65,103)$. Several studies suggest the importance of fucosylation associated with cancer and inflammation $(55,104)$. Fujita et al. investigated the association of urinary fucosylated PSA levels with the detection of aggressive PC (79). They investigated Lewistype or core-type fucosylated PSA (PSA-AAL) and coretype fucosylated PSA (PSA-PhoSL) in from urine in 69 patients who suspected PC (20 patients without PC and 49 patients with PC) and found urinary fucosylated PSA was significantly decreased in the men with $\mathrm{PC}$ compared with the men without $\mathrm{PC}(\mathrm{P}=0.026$ and $\mathrm{P}<0.001$, respectively). Also, both PSA-AAL and PSA-PhoSL were significantly associated with the Gleason scores of the biopsy specimens $(\mathrm{P}=0.001$, and $\mathrm{P}<0.001$, respectively). The area under the receiver-operator characteristic curve (AUC) value for the prediction of cancers of Gleason score $\geq 7$ was 0.69 
Table 1 Summary of urinary glycan biomarkers the information of the Food and Drug Administration (FDA) approved biomarkers and/or those commercially availability

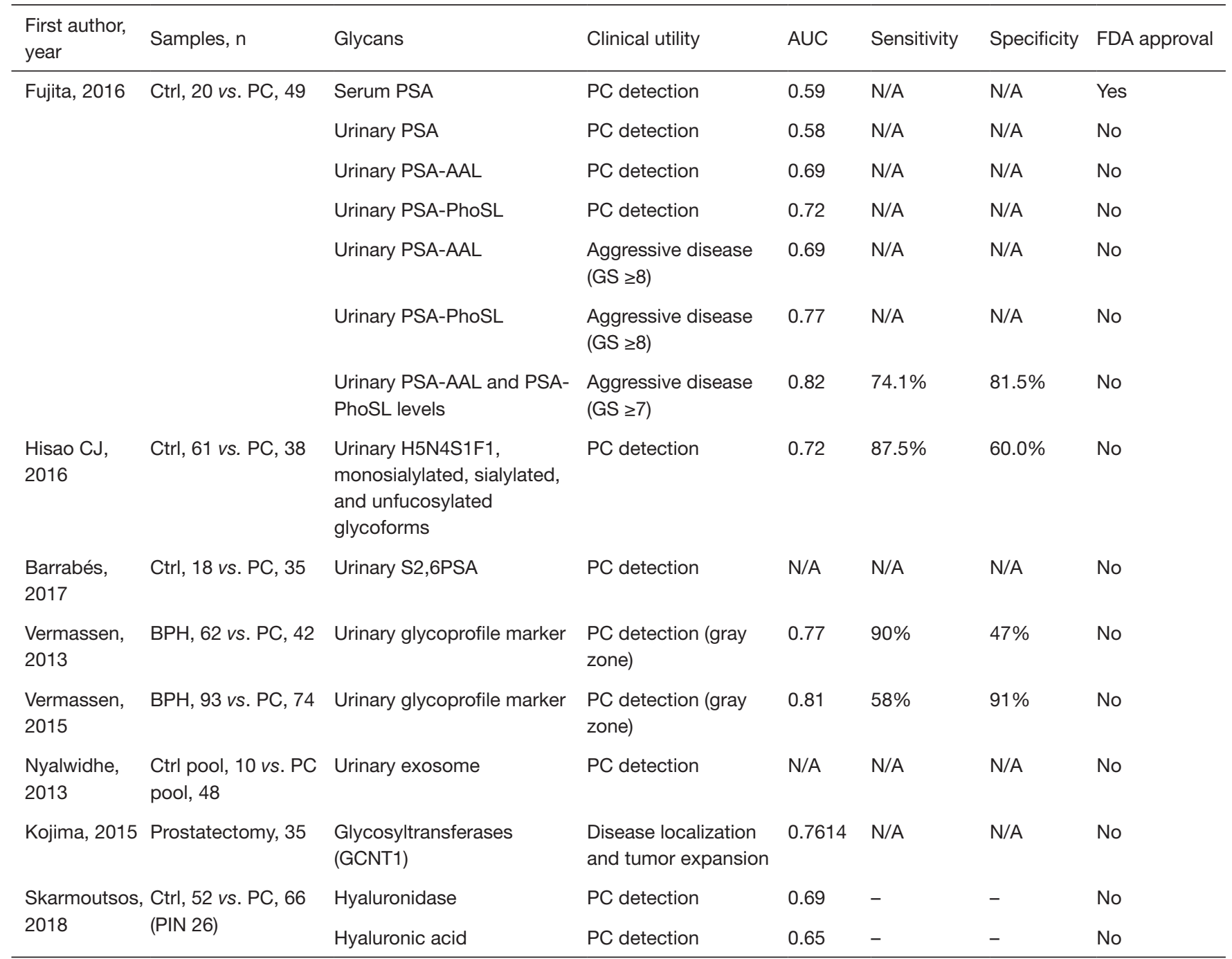

PIN, prostatic intraepithelial neoplasia ratio of nonfucosylated bi-, tri-, and tetra-antennary glycan structures on total of triantennary glycan structures; GCNT1, Core2 $\beta-1,6-N$-acetylglucosaminyltransferase-1; N/A, not applicable.

( $\mathrm{P}=0.0064)$ for urinary PSA-AAL and $0.72(\mathrm{P}=0.0014)$ for urinary PSA-PhoSL. They developed an optimum logistic regression model to predict the probability of detecting cancers with a GS $\geq 7$ in biopsy was obtained as $\mathrm{P}=[1+$ $\exp (1.247+4.56 \times$ PSAD $-0.00448 \times$ PSA-AAL -0.0493 $\times$ PSA-PhoSL)] -1 . Using this model, the AUC value for the prediction was $0.82(95 \%$ CI $0.72-0.92, \mathrm{P}<0.0001)$ with the sensitivity and specificity of the model at the best cutoff value were $74.1 \%$ and $81.5 \%$, respectively (Table 1 ). Although the biological mechanism leading to decreased urinary fucosylated PSA level in urine remains unclear, decreased urinary fucosylated PSA level may be a potential marker for aggressive PC.

It is possible to analyze urine PSA glycoforms using LC-MS with ion accumulation. Hsiao et al. reported that monosialylated, sialylated, and unfucosylated glycoforms of PSA were significantly different between PC and control samples (97). They investigated 61 benign prostate hyperplasia $(\mathrm{BPH})$ urine samples and $38 \mathrm{PC}$ urine samples. After the immunoprecipitation and in-gel protein 
digestions, the peptides and $\mathrm{N}$-glycopeptides generated from the chymotrypsin digestion were analyzed with an LC-MS. The normalized Hex5HexNAc4NeuAc1dHex1 (H5N4S1F1), monosialylated, sialylated, and unfucosylated glycoforms showed significant differences between $\mathrm{BPH}$ and PC. The ROC curve and the AUC of those glycoforms showed significant differences in PC detection with sensitivity and specificity of $87.5 \%$ and $60 \%$, respectively (Table 1). This result suggests the unfucosylated glycoforms of PSA were potential urinary glycan biomarkers in PC, in opposition to the results from Fujita et al. (79). One reason for this discrepancy might be the methodological differences between the lectin-antibody ELISA detection and LC-MS detection. Furthermore, the preparation of urine samples greatly influences the outcomes of downstream analyses. For example, urinary Tamm-Horsfall Protein (uromodulin) interferes with urinary assays and forms contaminant precipitates in the urine. Therefore, urinary aberrant PSA glycosylation needs further study to apply the clinical practice.

PSA has a single $N$-glycosylation site at asparagine-69 (103). Multiple studies have confirmed the ratio of complex biantennary glycans of $\alpha-2,3$-sialic acid (S2,3PSA) and $\alpha-2,6$-sialic acid (S2,6PSA) in serum have been closely linked to aggressive PC $(56,58,105,106)$ in over 50 glycoforms of serum PSA. However, this was not replicated in the urinary PSA. A previous study evaluated the clinical utility of S2,6PSA from urine after prostate massage in 35 patients diagnosed with PC and in 18 controls (98). They found no significant difference in S2,6PSA levels between the biopsy negative patients and PC patients with Gleason score $6(\mathrm{P}=0.364)$, between the biopsy negative patients and PC patients with Gleason score $7(\mathrm{P}=0.116)$, and between the biopsy negative patients and $\mathrm{PC}$ patents with Gleason score 8 or more $(\mathrm{P}=0.276)$. Also, they found no relationship was found between S2,6PSA and PC aggressiveness. These results may suggest the limited utility of S2,6PSA alone in urine to detect PC. The ratio of S2,3PSA and S2,6PSA needs to be investigated because these 2 glycoforms are associated with each other during the PC progression. Therefore, this finding needs to be interpreted with caution because of the small sample size and limitation measurement of PSA glycoforms. Currently, urinary fucosylated PSA levels are a promising biomarker for PC detection and aggressiveness among the aberrant PSA glycosylation.

\section{Capillary electrophoresis of urinary glycoproteins}

Capillary electrophoresis is a technique that separates molecules via an electric field according to size and charge. Several capillary electrophoresis-based systems for urinary glycan analysis are available, such as the Gly-Q system (Figure 4) and the multibacillary electrophoresis-based ABI3130 sequencer. Vermassen et al. (99) evaluated urinary $\mathrm{N}$-glycosylation profiles in post-prostate massage urine using capillary electrophoresis and demonstrated differences between patients with PC and benign prostate hyperplasia. Also, they developed a urinary glycoprofile marker (ratio of non-fucosylated bi-, tri-, and tetra-antennary glycan structures on total triantennary glycan structures divided by the prostate volume), and showed the potential to differentiate benign prostate hyperplasia from PC with the AUC, sensitivity, and specificity of $0.77,90 \%$, and $47 \%$, respectively (Table 1). The updated analysis showed similar performance of the urinary glycoprofile marker in the patients with a gray zone (Table 1). The predictive accuracy of the urinary glycoprofile marker was significantly better than that of serum PSA $(\mathrm{P}<0.001)(80)$. A Capillary electrophoresis system can analyze glycoprotein in urine; however, limited evidence is currently available. Also, we need to combine some glycans (such as urinary glycoprofile marker) to detect PC. Further large-scale studies are necessary to address the use of capillary electrophoresisbased analysis to identify urinary glycan PC biomarkers.

\section{Exosomes}

Exosomes are extracellular vesicles with a diameter of 30-200 $\mathrm{nm}$ that are secreted from most cell types. Urinary exosomes contain not only RNA and proteins, but also glycoproteins $(75,107)$. However, glycan profiles in exosomes are largely unexplored in patients with PC. Urinary exosomes in PC may be promising sources of novel biomarker discovery, as urinary glycoproteins can be analyzed by lectin-based ELISA, lectin-based microarray, capillary electrophoresis, and mass spectrometry. Nyalwidhe et al. (101) reported $N$-glycan profiling of urinary exosomes after prostate massage using lectin and MALDI-TOFbased profiling techniques. They demonstrated a decline of larger branched triantennary and tetraantennary $N$-glycans in exosomes using pooled samples (Table 1) (101). However, the major limitation of exosome analysis might be the 


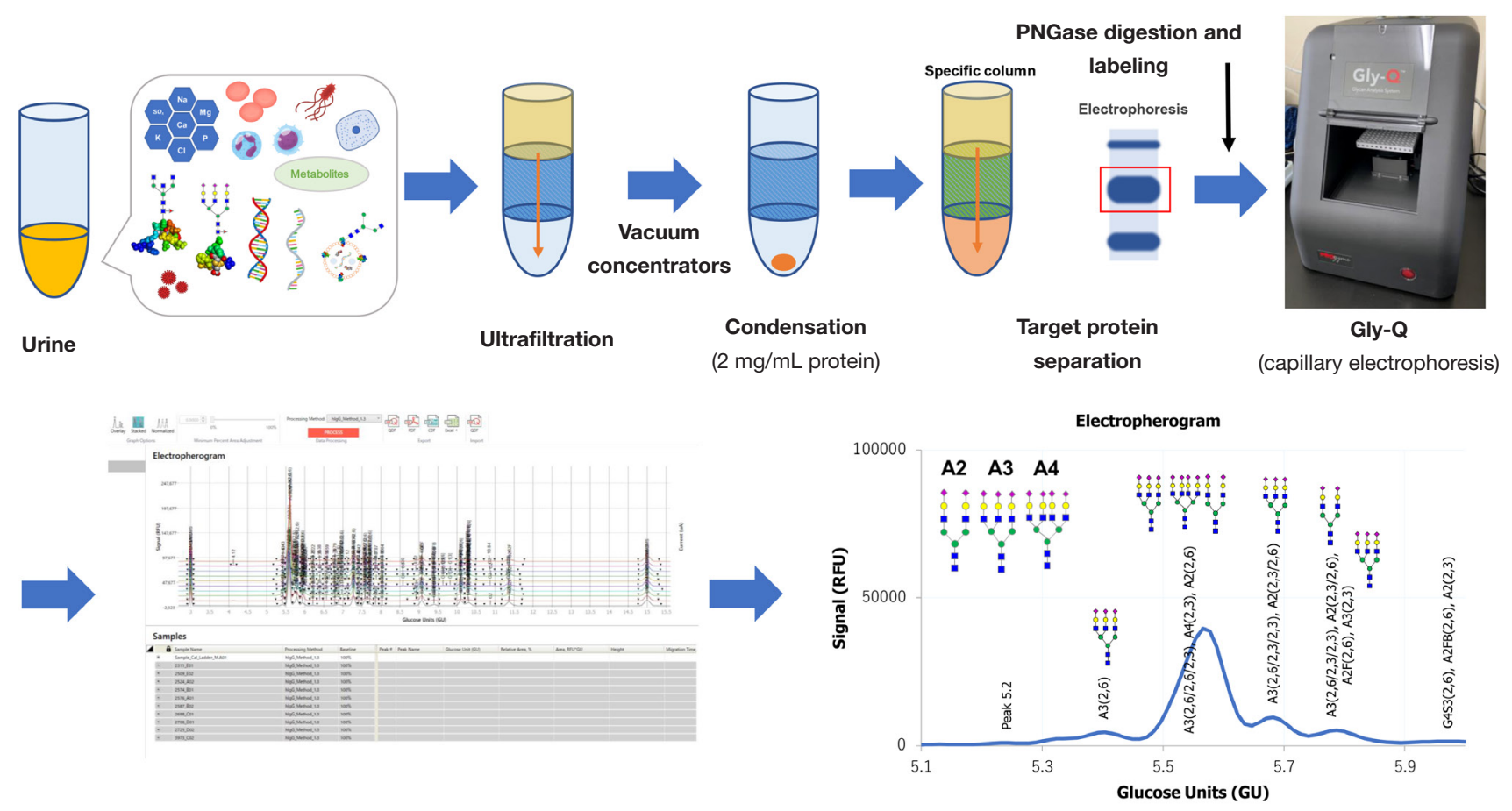

Figure 4 Schematic protocol of direct glycan analysis using capillary electrophoresis (Gly-Q). Capillary electrophoresis-LED-induced fluorescence-based Gly-Q N-glycan analysis system (Prozyme, Inc., CA, USA) combined with Gly-X rapid N-glycan preparation method can measure the amount of glycans under controlled automated SweetblotTM (System Instruments, Tokyo, Japan) machinery. Briefly, 1 mg/mL of target protein from the urine and $2 \mu \mathrm{L}$ of Gly-X denaturant was mixed. Then, $2 \mu \mathrm{L}$ of $N$-glycanase working solution was added to the denatured samples. After deglycosylation, $5 \mu \mathrm{L}$ of InstantPC dye solution was added to the deglycosylated samples. The InstantPC Dye and deglycosyalted sample mixture was then loaded onto prewetted Gly-X cleanup plate and applied vacuum to $<5$ inHg. Then, $100 \mu \mathrm{L}$ of Gly-X InstantPC eluent added to each well and collected InstanPC-labeled glycan samples into the Collection Plate using vacuum. Finally, InstantQ is a charged $N$-glycan dye that facilitates separation of labeled $N$-glycans on the Gly-Q CE system. Composition and structures of the glycans were analyzed using the Gly-Q Manager software performing automated peak analysis (Relative Fluorescence Unit: RFU and Glucose Unit: GU) and glycan assignments from the glycan library.

time-consuming methods of purification and small yield of exosomes from urine. Exosome preparation requires several sessions of ultracentrifugation (e.g., 25,000 $\times \mathrm{g}$ for $30 \mathrm{~min}$, followed by supernatant centrifugation at 100,000 $\times \mathrm{g}$ for $4 \mathrm{~h}$ ) and the expression of exosome protein markers (CD63 and CD9) needs to be verified. In some cases, pooled samples (from three patients) are needed due to small numbers of exosomes. Therefore, this protocol may not be feasible for the analysis of individual patients. Further methodological advancement for exosome enrichment is necessary for clinical application.

\section{Upregulation of glycosyltransferases}

Aberrant glycosylations are caused by the over- or under-expression of glycosyltransferases in cancer cells $(35,108)$. Several reports suggest positive links between aberrant glycosyltransferases and disease progression $(57,59,62,77,104,109-112)$ through the androgen receptor regulation. Of these, $\mathrm{N}$-acetylgalact osaminyltransferase 7 (GALNT7) and Core2 $\beta-1,6-\mathrm{N}-$ acetylglucosaminyltransferase-1 (GCNT1) are associated with androgen receptor splice variant-7 (AR-V7) (113). Taken together, these observations suggest the importance 
of glycosyltransferases for PC progression. However, only a few studies investigate the clinical utility of urinary glycosyltransferases in PC. Kojima et al. (77) have reported the detection of GCNT1 in post-massage urine by immunoblotting can predict the extracapsular extension of PC after radical prostatectomy. They investigated postdigital rectal examination urine from 35 patients before underwent radical prostatectomy and detected GCNT1 by an anti-GCNT1 monoclonal antibody, followed by a horseradish peroxidase (HRP)-conjugated antibody. The GCNT1 expression $(\mathrm{P}=0.006)$ was highly correlated to the extracapsular extension of $\mathrm{PC}$ in a logistic regression analysis with the AUC value of 0.7614 (Table 1). Of urinary glycan markers, GCNT1 may be a potential predictive marker for tumor recurrence after radical prostatectomy. However, the association of these glycosyltransferases with specific final products (glycans) remains unclear, as overexpression of individual glycosyltransferases does not always lead to the overexpression of specific glycans. Therefore, further studies are necessary before the use of these glycosyltransferases as diagnostic and prognostic biomarkers for PC.

\section{Hyaluronic acid and hyaluronidase}

Hyaluronic acid and hyaluronidase may represent potential novel urine biomarkers for the diagnosis of PC. Skarmoutsos et al. (100) investigated post-prostate massage urine from 118 high-risk PC patients, and hyaluronic acid and hyaluronidase were detected via enzyme-linked immunosorbent assay. Their results suggested that hyaluronic acid and hyaluronidase were independently associated with PC and that higher levels of hyaluronic acid and hyaluronidase were associated with a higher incidence of PC (100). ROC analysis for hyaluronic acid and hyaluronidase had a significant predictive ability for PC with AUC of 0.65 (70\% sensitivity and 55.2\% specificity) and 0.69 (65\% sensitivity and $53.9 \%$ specificity), respectively (Table 1). However, limitations of this study included the lack of molecular size analysis of hyaluronic acid and the determination of specific hyaluronidases. The molecular size of hyaluronic acid has been hypothesized to play a role in tumor aggressiveness $(114,115)$, and several types of hyaluronidases are associated with the digestion of hyaluronic acid $(116,117)$. Additionally, no information is available to correlate tumor aggressiveness with levels of hyaluronic acid and hyaluronidase. Therefore, these findings may suggest a role for hyaluronic acid and hyaluronidase, as yet understudied potential biomarkers for PC.

\section{The potential methodology of detection of urinary glycan biomarkers}

\section{Direct measurement of urinary glycans using mass spectrometry}

Advances in mass spectrometry has led to the direct detection of glycans from fluid-based samples. With this methodology, it is extremely important to purify glycans from contaminants despite small sample sizes. To achieve this, a new technology for glycan-specific enrichment, called "a glycoblotting method," was developed (96). A combination of glycoblotting and MALDI-TOF/MS enabled a high-throughput and quantitative glycomic analysis of various biological samples that included a large number of impurities (Figure 5) (44-46,49,51,60,118). However, the removal of impurities and adjustment of protein concentration is challenging for urinary analysis compared to a blood-based platform. Also, $O$-glycan analysis requires specialized techniques and processes to separate the $O$-glycans from proteins, such as a chemical reaction with ammonia salts. Although $\mathrm{N}$-glycans are able to be released from proteins by peptide $N$-glycanase-F or -A (PNGases), no enzyme can specifically release $O$-glycans from proteins. No study has evaluated $N$ - and $O$-glycans in urine from patients with PC, and only one study has evaluated urinary $O$-glycans in bladder cancer (44). The high cost of mass spectrometry is one of the major concerns in using this system for clinical applications. Further technological advancement is necessary for the direct measurement of urinary glycans.

\section{Lectin-based microarray analysis for glycoproteins}

Another methodology for the analysis of protein glycosylation is the lectin array (Figure 6). Lectins are glycan-binding proteins that selectively recognize free carbohydrates or glycoprotein epitopes. Lectin-based microarray systems have been developed to analyze both glycan profiles and glycoproteins $(60,95)$ and can analyze both serum and urine. Matsumoto et al. (60) reported the use of lectin-based microarrays to identify serum a-1acid glycoprotein in patients with metastatic castrationresistant PC (CRPC). They found terminal $\alpha-2,3$-sialylated glycan, $\alpha-2,6$-sialylated glycan, and terminal galactose were significantly increased in the CRPC patients (60). Anan et al. (95) reported the use of lectin-based microarrays to identify urinary osteopontin, and found that the glycosylation profile of osteopontin was significantly different in patients with urolithiasis (95). One limitation of 

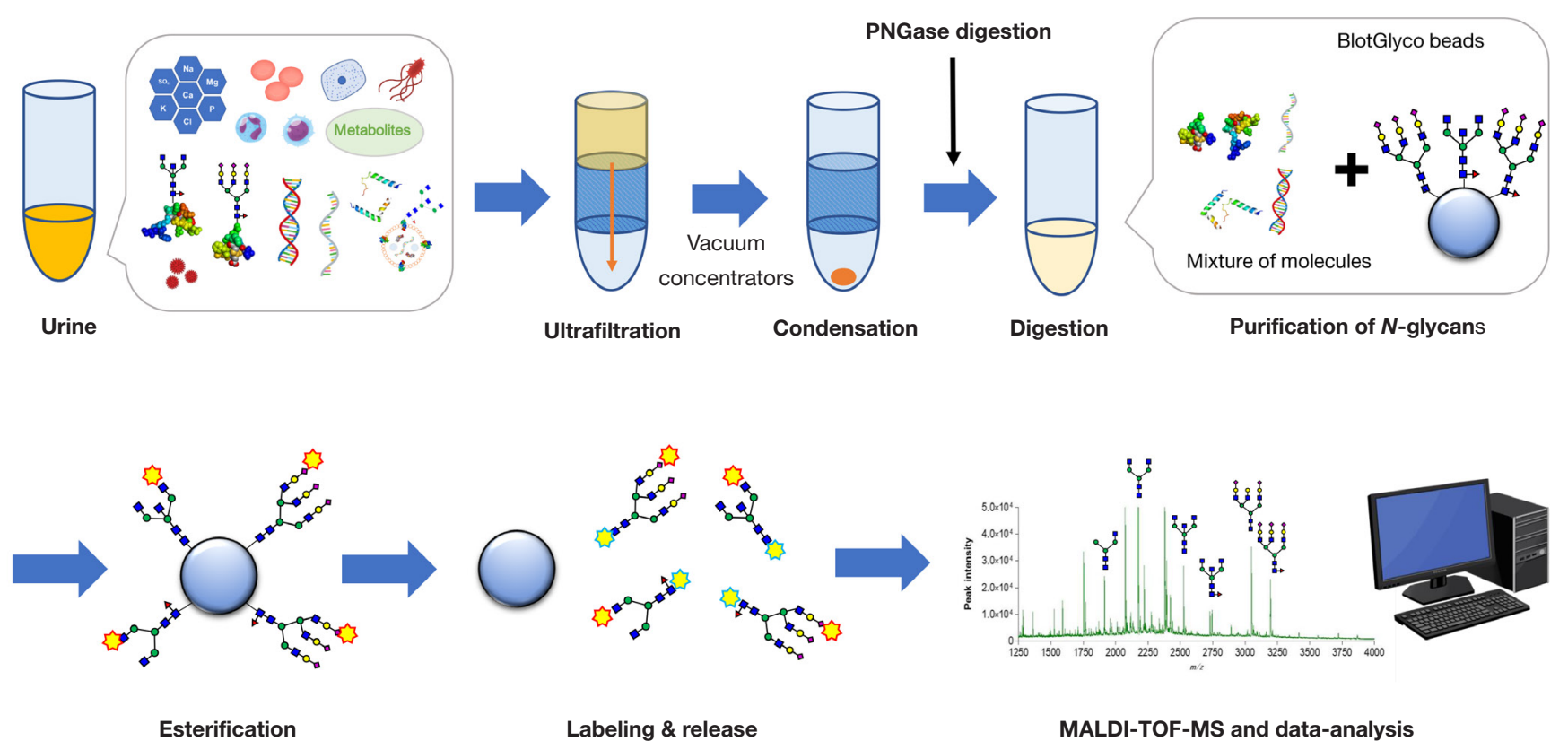

Figure 5 Schematic protocol of direct glycan analysis using SweetBlot and MALDI-TOF/MS. Fluid samples are applied to the SweetBlot for glycoblotting. After enzymatic cleavage from serum protein, total serum $\mathrm{N}$-glycans released into the digestion mixture are directly mixed with BlotGlyco H beads to capture N-glycans. After the beads are separated from other molecules by washing, sialic acid is methylesterified. These processed N-glycans are then labeled with benzyloxyamine (BOA) and released from BlotGlyco H beads. Mass spectra of BOA-labeled N-glycans are acquired using an Ultraflex III instrument.

this system is the requirement for protein concentration in urine samples. Concentrated urine $(2 \mathrm{mg} / \mathrm{mL}$ protein) was applied to a lectin-based microarray after ultrafiltration and vacuum concentration. As the density of urine varies in each sample, urinary protein concentrate needs to be adjusted and normalized for downstream analysis. Lectin-based microarray systems are promising methods of novel urinary biomarker discovery. However, no study has yet reported the use of lectin-based microarrays for urinary biomarkers in PC.

\section{Summary of urinary glycan biomarkers and the information of the Food and Drug Administration (FDA) approved biomarkers and/or those commercial availabilities}

In this narrative review, we showed potential urinary glycan biomarkers for PC detection and aggressive disease (Table 1). Of those, urinary fucosylated PSA levels are a promising biomarker for PC detection and aggressiveness among the aberrant PSA glycosylation. Urinary CGNT1 in the post-massage urine can be useful for the prediction of the extracapsular extension after radical prostatectomy. However, no FDA approved urinary glycan biomarker is available. Also, urinary glycan biomarker analyses were carried out using a custom technique, tools, and machines, while those are commercially available. Therefore, there is a significant hurdle between the urinary glycan analysis and clinical implementation. Therefore, urinary glycan analysis is far from clinical implementation. Further studies and methodological improvements are necessary to overcome these limitations.

\section{Conclusions}

Urinary glycan profiling exhibits high clinical potential as a noninvasive assay to monitor tumor heterogeneity and aggressiveness and may lead to personalized cancer therapies. Currently, urinary fucosylated PSA levels and urinary CGNT1 in the post-massage urine are a promising biomarker for PC detection and aggressiveness. Although several challenges remain, the technological development 


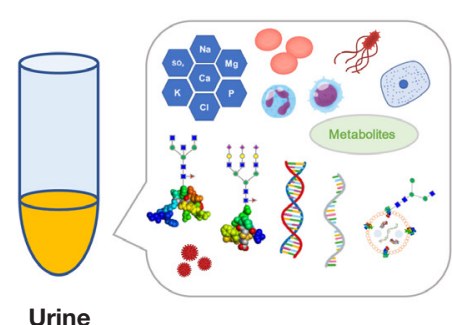

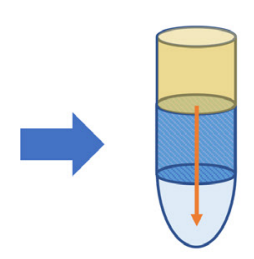

Ultrafiltration

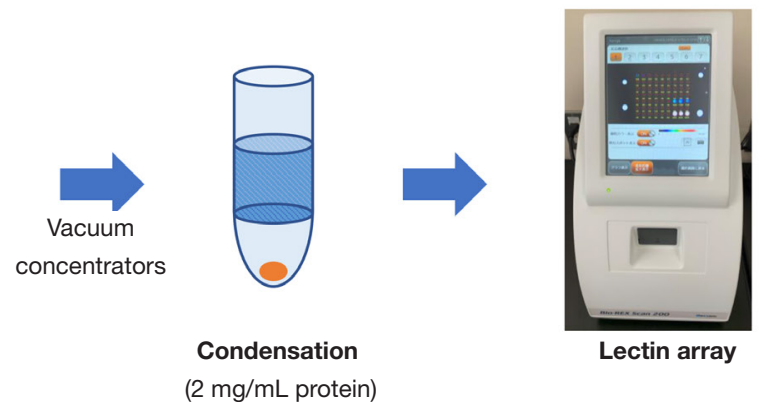

(2 $\mathrm{mg} / \mathrm{mL}$ protein)
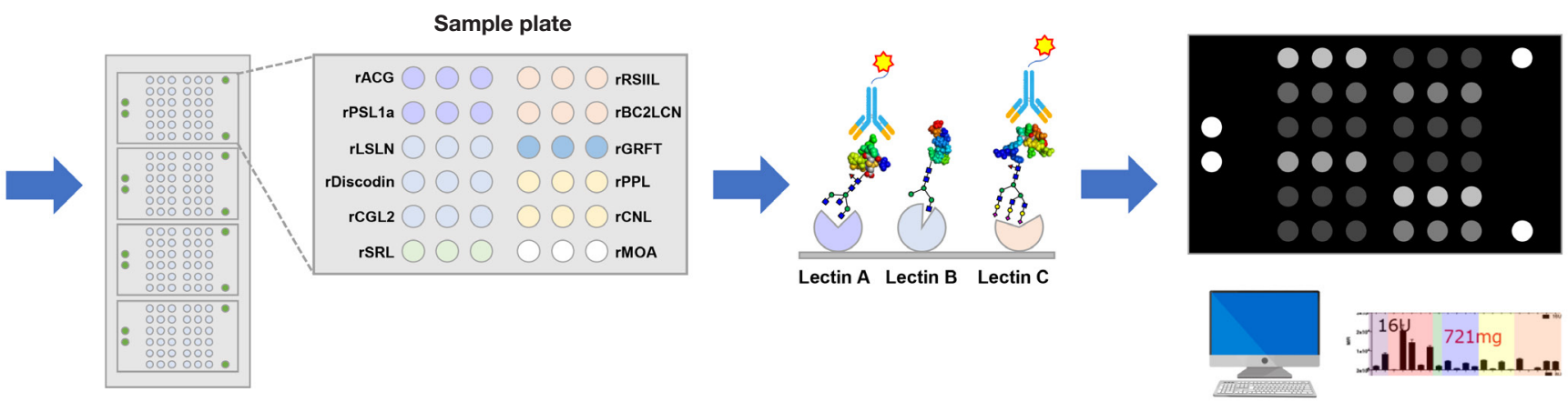

Figure 6 Schematic protocol of glycoprotein analysis using lectin-based microarray system. Glycoproteins are added to the wells of the recombinant lectin array chip. After reactions occur, the chip is scanned utilizing evanescent-wave fluorescence microscopy. Briefly, $20 \mu \mathrm{L}$ of sample was diluted by $80 \mu \mathrm{L}$ of probing buffer. Diluted sample was added to the well of recombinant lectin array chip (Rexxam Co. Ltd., Osaka, Japan). After 70 mins incubation and washing twice, $100 \mu \mathrm{L}$ of probing buffer containing $1 \mu \mathrm{g} / \mathrm{mL}$ biotinylated specific antibody for the target protein was added to well. After 60 mins incubation and washing twice, $100 \mu \mathrm{L}$ of probing buffer containing $1 \mu \mathrm{g} / \mathrm{mL} \mathrm{Cy} 3$ labeled streptavidin (GE-Healthcare, Buckinghamshire, UK) was added to the well. After 30 mins incubation and washing twice, the chip was scanned by utilizing Bio-REX Scan 200 evanescent fluorescence scanner (Rexxam Co., Ltd.). Mean fluorescence intensity of lectin reactive glycan carrying Igs was normalized by total Igs level.

of glycan analysis is rapidly advancing. Urinary glycan analysis is one of the most promising approaches for cancer biomarker discovery.

\section{Acknowledgments}

The authors would like to thank Yuki Fujita, Yukie Nishizawa, and Satomi Sakamoto for their invaluable support. The authors would also like to thank Enago (www. enago.jp) for English language review.

Funding: This work was supported by Japan Society for the Promotion of Science (JSPS) KAKENHI Grant Number 18K09157 (TY), 19H05556 (CO), and 20K09517 (SH).

\section{Footnote}

Provenance and Peer Review: This article was commissioned by the Guest Editor (Kazutoshi Fujita) for the series
"Urinary Biomarkers of Urothelial Malignancies" published in Translational Andrology and Urology. The article has undergone external peer review.

Reporting Checklist: The authors have completed the NARRATIVE REVIEW reporting checklist. Available at http://dx.doi.org/10.21037/tau-20-964

Peer Review File: Available at http://dx.doi.org/10.21037/ tau-20-964

Conflicts of Interest: All authors have completed the ICMJE uniform disclosure form (available at http://dx.doi. org/10.21037/tau-20-964). The series "Urinary Biomarkers of Urothelial Malignancies" was commissioned by the editorial office without any funding or sponsorship. The authors have no other conflicts of interest to declare.

Ethical Statement: the authors are accountable for all 
aspects of the work in ensuring that questions related to the accuracy or integrity of any part of the work are appropriately investigated and resolved. This study was performed according to the ethical standards of the Declaration of Helsinki and approved by the ethics review boards of the Hirosaki University School of Medicine (authorization number: 2019-001 and 2019-099).

Open Access Statement: This is an Open Access article distributed in accordance with the Creative Commons Attribution-NonCommercial-NoDerivs 4.0 International License (CC BY-NC-ND 4.0), which permits the noncommercial replication and distribution of the article with the strict proviso that no changes or edits are made and the original work is properly cited (including links to both the formal publication through the relevant DOI and the license). See: https://creativecommons.org/licenses/by-nc-nd/4.0/.

\section{References}

1. Chi KN, Agarwal N, Bjartell A, et al. Apalutamide for Metastatic, Castration-Sensitive Prostate Cancer. N Engl J Med 2019;381:13-24.

2. Davis ID, Martin AJ, Stockler MR, et al. Enzalutamide with Standard First-Line Therapy in Metastatic Prostate Cancer. N Engl J Med 2019;381:121-31.

3. Fizazi K, Tran N, Fein L, et al. Abiraterone plus Prednisone in Metastatic, Castration-Sensitive Prostate Cancer. N Engl J Med 2017;377:352-60.

4. James ND, de Bono JS, Spears MR, et al. Abiraterone for Prostate Cancer Not Previously Treated with Hormone Therapy. N Engl J Med 2017;377:338-51.

5. Culp MB, Soerjomataram I, Efstathiou JA, et al. Recent Global Patterns in Prostate Cancer Incidence and Mortality Rates. Eur Urol 2020;77:38-52.

6. Armstrong AJ, Szmulewitz RZ, Petrylak DP, et al. ARCHES: A Randomized, Phase III Study of Androgen Deprivation Therapy With Enzalutamide or Placebo in Men With Metastatic Hormone-Sensitive Prostate Cancer. J Clin Oncol 2019;37:2974-86.

7. Budnik J, Suri J, Bates JE, et al. Prognostic Significance of Sites of Visceral Metastatic Disease in Prostate Cancer: A Population-based Study of 12,180 Patients. Clin Genitourin Cancer 2019;17:260-7.

8. Oh WK, Miao R, Vekeman F, et al. Real-world Characteristics and Outcomes of Patients With Metastatic Castration-resistant Prostate Cancer Receiving Chemotherapy Versus Androgen Receptor-targeted
Therapy After Failure of First-line Androgen Receptortargeted Therapy in the Community Setting. Clin Genitourin Cancer 2017;16:50-7.

9. Narita S, Hatakeyama S, Takahashi M, et al. Clinical outcomes and prognostic factors in patients with newly diagnosed metastatic prostate cancer initially treated with androgen deprivation therapy: a retrospective multicenter study in Japan. Int J Clin Oncol 2020;25:912-20.

10. Mitsuzuka K, Arai Y. Metabolic changes in patients with prostate cancer during androgen deprivation therapy. Int J Urol 2018;25:45-53.

11. Momota M, Hatakeyama S, Soma O, et al. Geriatric 8 screening of frailty in patients with prostate cancer. Int $\mathrm{J}$ Urol 2020;27:642-8.

12. Murata Y, Tatsugami K, Yoshikawa M, et al. Predictive factors of biochemical recurrence after radical prostatectomy for high-risk prostate cancer. Int J Urol 2018;25:284-9.

13. Nakamura K, Konishi K, Komatsu T, et al. Quality of life after external beam radiotherapy for localized prostate cancer: Comparison with other modalities. Int J Urol 2019;26:950-4.

14. Okamoto T, Hatakeyama S, Narita S, et al. Validation and development of the CHAARTED criteria in patients with hormone-naive metastatic prostate cancer: A multiinstitutional retrospective study in Japan. Int J Urol 2020;27:90-1.

15. Saika T, Miura N, Fukumoto T, et al. Role of robotassisted radical prostatectomy in locally advanced prostate cancer. Int J Urol 2018;25:30-5.

16. Tilki D, Pompe RS, Bandini M, et al. Local treatment for metastatic prostate cancer: A systematic review. Int J Urol 2018;25:390-403.

17. Kato T, Sugimoto M. Quality of life in active surveillance for early prostate cancer. Int J Urol 2020;27:296-306.

18. Kijima T, Ito M, Ishioka J, et al. Treatment-induced changes in levels of brain natriuretic peptide in prostate cancer patients receiving gonadotropin-releasing hormone antagonists or agonists. Int J Urol 2020;27:354-5.

19. Fujita N, Koie T, Hashimoto Y, et al. Neoadjuvant chemohormonal therapy followed by robot-assisted and minimum incision endoscopic radical prostatectomy in patients with high-risk prostate cancer: comparison of perioperative and oncological outcomes at single institution. Int Urol Nephrol 2018;50:1999-2005.

20. Hagiwara K, Koie T, Ohyama C, et al. Efficacy of a neoadjuvant gonadotropin-releasing hormone antagonist plus low-dose estramustine phosphate in high-risk 
prostate cancer: a single-center study. Int Urol Nephrol 2017;49:811-6.

21. Crawford ED, Grubb R, 3rd, Black A, et al. Comorbidity and mortality results from a randomized prostate cancer screening trial. J Clin Oncol 2011;29:355-61.

22. Basu A, Gore JL. Are Elderly Patients With Clinically Localized Prostate Cancer Overtreated? Exploring Heterogeneity in Survival Effects. Med Care 2015;53:79-86.

23. Matsumoto T, Hatakeyama S, Ookubo T, et al. Costeffectiveness comparison between neoadjuvant chemohormonal therapy and extended pelvic lymph node dissection in high-risk prostate cancer patients treated with radical prostatectomy: a multi-institutional analysis. Med Oncol 2017;34:190.

24. Narita T, Koie T, Ookubo T, et al. The impact of extended lymph node dissection versus neoadjuvant therapy with limited lymph node dissection on biochemical recurrence in high-risk prostate cancer patients treated with radical prostatectomy: a multi-institutional analysis. Med Oncol 2017;34:1.

25. Ito K, Oki R, Sekine Y, et al. Screening for prostate cancer: History, evidence, controversies and future perspectives toward individualized screening. Int J Urol 2019;26:956-70.

26. Kakehi Y, Sugimoto M, Taoka R. Evidenced-based clinical practice guideline for prostate cancer Int J Urol 2017;24:648-66.

27. Kimura T, Egawa S. Epidemiology of prostate cancer in Asian countries. Int J Urol 2018;25:524-31.

28. Komura K, Sweeney CJ, Inamoto T, et al. Current treatment strategies for advanced prostate cancer. Int J Urol 2018;25:220-31.

29. Kodama H, Hatakeyama S, Narita S, et al. Clinical Characterization of Low Prostate-specific Antigen on Prognosis in Patients With Metastatic Castrationnaive Prostate Cancer. Clin Genitourin Cancer 2019;17:e1091-8.

30. Mori K, Kimura S, Parizi MK, et al. Prognostic Value of Lactate Dehydrogenase in Metastatic Prostate Cancer: A Systematic Review and Meta-analysis. Clin Genitourin Cancer 2019;17:409-18.

31. Okita K, Hatakeyama S, Narita S, et al. The Effect of Treatment Sequence on Overall Survival for Men With Metastatic Castration-resistant Prostate Cancer: A Multicenter Retrospective Study. Clin Genitourin Cancer 2020;18:e103-11.

32. Akamatsu S, Kubota M, Uozumi R, et al. Development and Validation of a Novel Prognostic Model for Predicting Overall Survival in Treatment-naive Castration-sensitive Metastatic Prostate Cancer. Eur Urol Oncol 2019;2:320-8.

33. Hamano I, Hatakeyama S, Narita S, et al. Impact of nadir PSA level and time to nadir during initial androgen deprivation therapy on prognosis in patients with metastatic castration-resistant prostate cancer. World J Urol 2019;37:2365-73.

34. Okamoto T, Hatakeyama S, Narita S, et al. Impact of nutritional status on the prognosis of patients with metastatic hormone-naive prostate cancer: a multicenter retrospective cohort study in Japan. World J Urol 2019;37:1827-35.

35. Hatakeyama S, Yoneyama T, Tobisawa Y, et al. Recent progress and perspectives on prostate cancer biomarkers. Int J Clin Oncol 2017;22:214-21.

36. Yoshida S, Takahara T, Arita Y, et al. Patterns of failure after progressive site-directed therapy in oligoprogressive castration-resistant prostate cancer. Int J Urol 2020;27:634-5.

37. Chi KN, Annala M, Sunderland K, et al. A randomized phase II cross-over study of abiraterone + prednisone (ABI) vs enzalutamide (ENZ) for patients (pts) with metastatic, castration-resistant prostate cancer (mCRPC). J Clin Oncol 2017;35:5002.

38. Zhang Z, Wuhrer M, Holst S. Serum sialylation changes in cancer. Glycoconj J 2018;35:139-60.

39. Kizuka Y, Funayama S, Shogomori H, et al. HighSensitivity and Low-Toxicity Fucose Probe for Glycan Imaging and Biomarker Discovery. Cell Chem Biol 2016;23:782-92.

40. Stanley P, Taniguchi N, Aebi M. N-Glycans. In: Varki A, Cummings RD, Esko JD et al. (eds). Essentials of Glycobiology. Cold Spring Harbor (NY): Cold Spring Harbor Laboratory Press, 2015:99-111.

41. Harada Y, Ohkawa Y, Kizuka Y, et al. Oligosaccharyltransferase: A Gatekeeper of Health and Tumor Progression. Int J Mol Sci 2019;20:6074.

42. Carvalho S, Oliveira T, Bartels MF, et al. O-mannosylation and N-glycosylation: two coordinated mechanisms regulating the tumour suppressor functions of E-cadherin in cancer. Oncotarget 2016;7:65231-46.

43. Mereiter S, Balmana M, Campos D, et al. Glycosylation in the Era of Cancer-Targeted Therapy: Where Are We Heading? Cancer Cell 2019;36:6-16.

44. Takeuchi M, Amano M, Kitamura H, et al. N- and O-glycome analysis of serum and urine from bladder cancer patients using a high-throughput glycoblotting 
method. J Glycomics Lipidomics 2013;3:1.

45. Hatakeyama S, Amano M, Tobisawa Y, et al. Serum $\mathrm{N}$-glycan alteration associated with renal cell carcinoma detected by high throughput glycan analysis. J Urol 2014;191:805-13.

46. Hatakeyama S, Amano M, Tobisawa Y, et al. Serum $\mathrm{N}$-glycan profiling predicts prognosis in patients undergoing hemodialysis. ScientificWorldJournal 2013;2013:268407.

47. Oikawa M, Hatakeyama S, Yoneyma T, et al. Significance of Serum N-glycan Profiling as a Diagnostic Biomarker in Urothelial Carcinoma. Eur Urol Focus 2018;4:405-11.

48. Narita S, Nomura K, Hatakeyama S, et al. Changes in conditional net survival and dynamic prognostic factors in patients with newly diagnosed metastatic prostate cancer initially treated with androgen deprivation therapy. Cancer Med 2019;8:6566-77.

49. Noro D, Yoneyama T, Hatakeyama S, et al. Serum Aberrant N-Glycan Profile as a Marker Associated with Early Antibody-Mediated Rejection in Patients Receiving a Living Donor Kidney Transplant. Int J Mol Sci 2017;18:1731.

50. Yoneyama T, Hatakeyama S, Tobisawa Y, et al. Blood group antigen-targeting peptide suppresses anti-blood group antibody binding to antigen in renal glomerular capillaries after ABO-incompatible blood reperfusion. Transplantation 2013;95:418-25.

51. Soma O, Hatakeyama S, Yoneyama T, et al. Serum $\mathrm{N}$-glycan profiling can predict biopsy-proven graft rejection after living kidney transplantation. Clin Exp Nephrol 2020;24:174-84.

52. Tanaka T, Yoneyama T, Noro D, et al. Aberrant N-Glycosylation Profile of Serum Immunoglobulins is a Diagnostic Biomarker of Urothelial Carcinomas. Int J Mol Sci 2017;18:2632.

53. Taniguchi N, Kizuka Y. Glycans and cancer: role of $\mathrm{N}$-glycans in cancer biomarker, progression and metastasis, and therapeutics. Adv Cancer Res 2015;126:11-51.

54. Kyselova Z, Mechref Y, Al Bataineh MM, et al. Alterations in the serum glycome due to metastatic prostate cancer. J Proteome Res 2007;6:1822-32.

55. Fujita K, Shimomura M, Uemura M, et al. Serum fucosylated haptoglobin as a novel prognostic biomarker predicting high-Gleason prostate cancer. Prostate 2014;74:1052-8.

56. Yoneyama T, Ohyama C, Hatakeyama S, et al. Measurement of aberrant glycosylation of prostate specific antigen can improve specificity in early detection of prostate cancer. Biochem Biophys Res Commun 2014;448:390-6.

57. Mikami J, Tobisawa Y, Yoneyama T, et al. I-branching $\mathrm{N}$-acetylglucosaminyltransferase regulates prostate cancer invasiveness by enhancing alpha5beta 1 integrin signaling. Cancer Sci 2016;107:359-68.

58. Yoneyama T, Tobisawa Y, Kaneko T, et al. Clinical significance of the LacdiNAc-glycosylated prostate-specific antigen assay for prostate cancer detection. Cancer Sci 2019;110:2573-89.

59. Ishibashi Y, Tobisawa Y, Hatakeyama S, et al. Serum triand tetra-antennary $\mathrm{N}$-glycan is a potential predictive biomarker for castration-resistant prostate cancer. Prostate 2014;74:1521-9.

60. Matsumoto T, Hatakeyama S, Yoneyama T, et al. Serum $\mathrm{N}$-glycan profiling is a potential biomarker for castrationresistant prostate cancer. Sci Rep 2019;9:16761.

61. Hagisawa S, Ohyama C, Takahashi T, et al. Expression of core 2 beta1,6-N-acetylglucosaminyltransferase facilitates prostate cancer progression. Glycobiology 2005;15:1016-24.

62. Hagiwara K, Tobisawa Y, Kaya T, et al. Wisteria floribunda Agglutinin and Its Reactive-Glycan-Carrying ProstateSpecific Antigen as a Novel Diagnostic and Prognostic Marker of Prostate Cancer. Int J Mol Sci 2017;18:261.

63. Ohyama C, Hosono M, Nitta K, et al. Carbohydrate structure and differential binding of prostate specific antigen to Maackia amurensis lectin between prostate cancer and benign prostate hypertrophy. Glycobiology 2004;14:671-9.

64. Prakash S, Robbins PW. Glycotyping of prostate specific antigen. Glycobiology 2000;10:173-6.

65. Tajiri M, Ohyama C, Wada Y. Oligosaccharide profiles of the prostate specific antigen in free and complexed forms from the prostate cancer patient serum and in seminal plasma: a glycopeptide approach. Glycobiology 2008;18:2-8.

66. Dos Santos Silva PM, Albuquerque PBS, de Oliveira WF, et al. Glycosylation products in prostate diseases. Clin Chim Acta 2019;498:52-61.

67. Saldova R, Fan Y, Fitzpatrick JM, et al. Core fucosylation and alpha2-3 sialylation in serum $\mathrm{N}$-glycome is significantly increased in prostate cancer comparing to benign prostate hyperplasia. Glycobiology 2011;21:195-205.

68. Clark DJ, Schnaubelt M, Hoti N, et al. Impact of Increased FUT8 Expression on the Extracellular Vesicle Proteome in Prostate Cancer Cells. J Proteome Res 
2020;19:2195-205.

69. Lang R, Rolny V, Leinenbach A, et al. Investigation on core-fucosylated prostate-specific antigen as a refined biomarker for differentiation of benign prostate hyperplasia and prostate cancer of different aggressiveness. Tumour Biol 2019;41:1010428319827223.

70. Premaratne P, Welén K, Damber JE, et al. O-glycosylation of MUC1 mucin in prostate cancer and the effects of its expression on tumor growth in a prostate cancer xenograft model. Tumour Biol 2011;32:203-13.

71. Scott E, Munkley J. Glycans as Biomarkers in Prostate Cancer. Int J Mol Sci 2019;20:1389.

72. Konety BR, Getzenberg RH. Urine based markers of urological malignancy. J Urol 2001;165:600-11.

73. Belczacka I, Pejchinovski M, Krochmal M, et al. Urinary Glycopeptide Analysis for the Investigation of Novel Biomarkers. Proteomics Clin Appl 2019;13:e1800111.

74. Jia G, Dong Z, Sun C, et al. Alterations in expressed prostate secretion-urine PSA N-glycosylation discriminate prostate cancer from benign prostate hyperplasia.

Oncotarget 2017;8:76987-99.

75. Fujita K, Nonomura N. Urinary biomarkers of prostate cancer. Int J Urol 2018;25:770-9.

76. Fujita K, Kume H, Matsuzaki K, et al. Proteomic analysis of urinary extracellular vesicles from high Gleason score prostate cancer. Sci Rep 2017;7:42961.

77. Kojima Y, Yoneyama T, Hatakeyama S, et al. Detection of Core2 beta-1,6-N-Acetylglucosaminyltransferase in PostDigital Rectal Examination Urine Is a Reliable Indicator for Extracapsular Extension of Prostate Cancer. PLoS One 2015; 10:e0138520.

78. Vermassen T, D'Herde K, Jacobus D, et al. Release of urinary extracellular vesicles in prostate cancer is associated with altered urinary $\mathrm{N}$-glycosylation profile. J Clin Pathol 2017;70:838-46.

79. Fujita K, Hayashi T, Matsuzaki K, et al. Decreased fucosylated PSA as a urinary marker for high Gleason score prostate cancer. Oncotarget 2016;7:56643-9.

80. Vermassen T, Van Praet C, Lumen N, et al. Urinary prostate protein glycosylation profiling as a diagnostic biomarker for prostate cancer. Prostate 2015;75:314-22.

81. Valverde P, Ardá A, Reichardt NC, et al. Glycans in drug discovery. Medchemcomm 2019;10:1678-91.

82. Brockhausen I, Schachter H, Stanley P. O-GalNAc Glycans. In: Varki A, Cummings RD, Esko JD et al., editors. Essentials of Glycobiology. Cold Spring Harbor (NY): Cold Spring Harbor Laboratory Press, 2009.

83. Chang YH, Weng CL, Lin KI. O-GlcNAcylation and its role in the immune system. J Biomed Sci 2020;27:57.

84. Gupta R, Leon F, Rauth S, et al. A Systematic Review on the Implications of O-linked Glycan Branching and Truncating Enzymes on Cancer Progression and Metastasis. Cells 2020;9:446.

85. Urata Y, Takeuchi H. Effects of Notch glycosylation on health and diseases. Dev Growth Differ 2020;62:35-48.

86. Costa AF, Campos D, Reis CA, et al. Targeting Glycosylation: A New Road for Cancer Drug Discovery. Trends Cancer 2020;6:757-66.

87. McKiernan J, Donovan MJ, O'Neill V, et al. A Novel Urine Exosome Gene Expression Assay to Predict Highgrade Prostate Cancer at Initial Biopsy. JAMA Oncol 2016;2:882-9.

88. Cucchiara V, Cooperberg MR, Dall'Era M, et al. Genomic Markers in Prostate Cancer Decision Making. Eur Urol 2018;73:572-82.

89. Holland B, Karr M, Delfino K, et al. The effect of the urinary and faecal microbiota on lower urinary tract symptoms measured by the International Prostate Symptom Score: analysis utilising next-generation sequencing. BJU Int 2020;125:905-10.

90. Xie J, Huang JS, Huang XJ, et al. Profiling the urinary microbiome in men with calcium-based kidney stones. BMC Microbiol 2020;20:41.

91. Gerges-Knafl D, Pichler P, Zimprich A, et al. The urinary microbiome shows different bacterial genera in renal transplant recipients and non-transplant patients at time of acute kidney injury - a pilot study. BMC Nephrol 2020;21:117.

92. Chen YB, Hochstedler B, Pham TT, et al. The Urethral Microbiota: A Missing Link in the Female Urinary Microbiota. J Urol 2020;204:303-9.

93. Aubin SM, Reid J, Sarno MJ, et al. PCA3 molecular urine test for predicting repeat prostate biopsy outcome in populations at risk: validation in the placebo arm of the dutasteride REDUCE trial. J Urol 2010;184:1947-52.

94. Tutrone R, Donovan MJ, Torkler P, et al. Clinical utility of the exosome based ExoDx Prostate(IntelliScore) EPI test in men presenting for initial Biopsy with a PSA 2-10 ng/ mL. Prostate Cancer Prostatic Dis 2020;23:607-14.

95. Anan G, Yoneyama T, Noro D, et al. The Impact of Glycosylation of Osteopontin on Urinary Stone Formation. Int J Mol Sci 2019;21:93.

96. Nishimura S, Niikura K, Kurogochi M, et al. Highthroughput protein glycomics: combined use of chemoselective glycoblotting and MALDI-TOF/ TOF mass spectrometry. Angew Chem Int Ed Engl 
2004;44:91-6.

97. Hsiao CJ, Tzai TS, Chen CH, et al. Analysis of Urinary Prostate-Specific Antigen Glycoforms in Samples of Prostate Cancer and Benign Prostate Hyperplasia. Dis Markers 2016;2016:8915809.

98. Barrabés S, Llop E, Ferrer-Batallé M, et al. Analysis of urinary PSA glycosylation is not indicative of high-risk prostate cancer. Clin Chim Acta 2017;470:97-102.

99. Vermassen T, Van Praet C, Vanderschaeghe D, et al. Capillary electrophoresis of urinary prostate glycoproteins assists in the diagnosis of prostate cancer. Electrophoresis 2014;35:1017-24.

100.Skarmoutsos I, Skarmoutsos A, Katafigiotis I, et al. Hyaluronic acid and hyaluronidase as possible novel urine biomarkers for the diagnosis of prostate cancer. Med Oncol 2018;35:97.

101. Nyalwidhe JO, Betesh LR, Powers TW, et al. Increased bisecting $\mathrm{N}$-acetylglucosamine and decreased branched chain glycans of $\mathrm{N}$-linked glycoproteins in expressed prostatic secretions associated with prostate cancer progression. Proteomics Clin Appl 2013;7:677-89.

102. Hongo F, Okihara K, Kitamura K, et al. Prostate cancer meeting the Japanese active surveillance criteria and diagnosed by community-based prostate-specific antigen screening: A 21-year follow-up study. Int J Urol 2019;26:827-32.

103. Drake RR, Jones EE, Powers TW, et al. Altered glycosylation in prostate cancer. Adv Cancer Res 2015;126:345-82.

104.Miyoshi E, Moriwaki K, Nakagawa T. Biological function of fucosylation in cancer biology. J Biochem 2008;143:725-9.

105.Ferrer-Batallé M, Llop E, Ramírez M, et al. Comparative Study of Blood-Based Biomarkers, $\alpha 2,3$-Sialic Acid PSA and PHI, for High-Risk Prostate Cancer Detection. Int J Mol Sci 2017;18:845.

106. Ishikawa T, Yoneyama T, Tobisawa Y, et al. An Automated Micro-Total Immunoassay System for Measuring CancerAssociated alpha2,3-linked Sialyl N-Glycan-Carrying Prostate-Specific Antigen May Improve the Accuracy of Prostate Cancer Diagnosis. Int J Mol Sci 2017;18:470.

Cite this article as: Hatakeyama S, Yoneyama T, Tobisawa Y, Yamamoto H, Ohyama C. Narrative review of urinary glycan biomarkers in prostate cancer. Transl Androl Urol 2021;10(4):18501864. doi: 10.21037/tau-20-964
107. Williams C, Royo F, Aizpurua-Olaizola O, et al. Glycosylation of extracellular vesicles: current knowledge, tools and clinical perspectives. J Extracell Vesicles 2018;7:1442985.

108.Häuselmann I, Borsig L. Altered tumor-cell glycosylation promotes metastasis. Front Oncol 2014;4:28.

109. Munkley J. Glycosylation is a global target for androgen control in prostate cancer cells. Endocr Relat Cancer 2017;24:R49-64.

110. Munkley J, Vodak D, Livermore KE, et al. Glycosylation is an Androgen-Regulated Process Essential for Prostate Cancer Cell Viability. EBioMedicine 2016;8:103-16.

111. Sato T, Yoneyama T, Tobisawa Y, et al. Core $2 \beta-1,6-\mathrm{N}$-ac etylglucosaminyltransferase-1 expression in prostate biopsy specimen is an indicator of prostate cancer aggressiveness. Biochem Biophys Res Commun 2016;470:150-6.

112.Li J, Guillebon AD, Hsu JW, et al. Human fucosyltransferase 6 enables prostate cancer metastasis to bone. Br J Cancer 2013;109:3014-22.

113. Antonarakis ES, Lu C, Wang H, et al. AR-V7 and Resistance to Enzalutamide and Abiraterone in Prostate Cancer. N Engl J Med 2014;371:1028-38.

114. Khaldoyanidi SK, Goncharova V, Mueller B, et al. Hyaluronan in the healthy and malignant hematopoietic microenvironment. Adv Cancer Res 2014;123:149-89.

115. Tavianatou AG, Caon I, Franchi M, et al. Hyaluronan: molecular size-dependent signaling and biological functions in inflammation and cancer. Febs $\mathrm{j}$ 2019;286:2883-908.

116. Yamaguchi Y, Yamamoto H, Tobisawa Y, et al. TMEM2: A missing link in hyaluronan catabolism identified? Matrix Biol 2019;78-79:139-46.

117. Yamamoto H, Tobisawa Y, Inubushi T, et al. A mammalian homolog of the zebrafish transmembrane protein 2 (TMEM2) is the long-sought-after cell-surface hyaluronidase. J Biol Chem 2017;292:7304-13.

118. Narita T, Hatakeyama S, Yoneyama T, et al. Clinical implications of serum $\mathrm{N}$-glycan profiling as a diagnostic and prognostic biomarker in germ-cell tumors. Cancer Med 2017;6:739-48. 